OPEN ACCESS

Edited by:

Ivano Alessandri,

University of Brescia, Italy

Reviewed by:

Aihua Liu,

Qingdao University, China

Nicolò Bontempi,

Catholic University of the

Sacred Heart, Italy

*Correspondence:

Zhenglong Zhang

zlzhang@snnu.edu.cn

Hairong Zheng

hrzheng@snnu.edu.cn

†These authors have contributed equally to this work

Specialty section

This article was submitted to Analytical Chemistry,

a section of the journal

Frontiers in Chemistry

Received: 19 April 2019 Accepted: 10 September 2019

Published: 24 September 2019

Citation:

Zhang $C$, Zhang T, Zhang $Z$ and Zheng H (2019) Plasmon Enhanced Fluorescence and Raman Scattering by [Au-Ag Alloy NP Cluster]@SiO 2

Core-Shell Nanostructure. Front. Chem. 7:647.

doi: 10.3389/fchem.2019.00647

\section{Plasmon Enhanced Fluorescence and Raman Scattering by [Au-Ag Alloy NP Cluster]@SiO 2 Core-Shell Nanostructure}

\author{
Chengyun Zhang ${ }^{\dagger}$, Tingting Zhang ${ }^{\dagger}$, Zhenglong Zhang ${ }^{*}$ and Hairong Zheng* \\ School of Physics and Information Technology, Shaanxi Normal University, Xi'an, China
}

Silica-shell coated noble metal nanoparticles have shown a good performance in surface enhanced fluorescence and Raman scattering. However, silica-shell coated single noble nanoparticle cannot effectively enhance the optical signal due to the relative weak near-field enhancement. In this paper, [Au-Ag alloy NP cluster]@SiO 2 core-shell nanostructure is employed to achieve the effective electric field enhancement. With the specific structure, simultaneous Raman scattering and fluorescence emission enhancement is obtained, and the enhancement comparison of fluorescence emission with Raman scattering in different type agglomeration of metal NPs is investigated in-situ. With different thickness of $\mathrm{SiO}_{2}$ shell, the optimized Raman and fluorescence enhancement systems are obtained, respectively, and corresponding study of power dependence are investigated in detail. The selectively enhanced Raman and fluorescence can be realized via controlling the shell thickness and laser power. Our work provides a non-polarization dependent [metal NP cluster]@ $\mathrm{SiO}_{2}$ system, which may have a promising application in portable chemical and biochemistry detecting.

Keywords: plasmon enhanced spectroscopy, raman scattering, fluorescence, core-shell structure, Au-Ag alloy NP cluster

\section{INTRODUCTION}

Nobel metal based nanoparticles (NPs), such as silver or gold NPs, have shown a great potential in plasmon sensor (Homola et al., 1999; Chen and Ming, 2012; Rosman et al., 2013), catalyst (Christopher et al., 2011; Han et al., 2015; Vadai et al., 2018; Zhang et al., 2018), biotherapy (Wang et al., 2014), and signal enhancement (Li et al., 2010; Ando et al., 2011; Wang and Kong, 2015) because of the abundant tunable surface plasmon resonance (SPR) peak in wide-range spectrum. The position of the SPR peak can be easily controlled through changing the morphology and material of the NPs (Liu et al., 2017). However, for pure Ag or Au NPs, it is hard to manipulate the SPR wavelength, especially at the wavelength of 400-530 nm (Liu et al., 2011; Li et al., 2017), which limits its application to some extent. Bimetallic Au-Ag alloy NPs provide a solution to the fault of the SPR wavelength, which can keep the morphology and size of metal NPs unchanged (Kuladeep et al., 2012; Rioux et al., 2014). Moreover, the Au-Ag alloy has congregated the advantages of Au and Ag, such as high chemical stability (Gao et al., 2014) and good photocatalytic properties (Tsukamoto et al., 2012; Han et al., 2016), and it is considered to be a promising candidate in metal material.

For years, many researches have been focused on plasmonic NPs enhancement of the optical signal (Ming et al., 2009; Schietinger et al., 2010; Ding et al., 2016), which is utilizing the near-field 
generated by the plasmonic NPs. The property of the Electricmagnetic (EM) field generated by the NPs is highly dependent on the morphology of the NPs and the features of the incident light (Sherry et al., 2005; Talley et al., 2005; Hao et al., 2007). For certain isolated NP structures, the enhancement of the near-field is highly dependent on the polarization of the incident light and the size of the NPs (Nie and Emory, 1997; Mia et al., 2019), and the effect of the enhancement is relatively lower compared with the cluster structure. For the cluster structure, the aggregation of the small particles generates lots of the hot spot, which effectively improves the intensity of the surrounding EM field (Adams et al., 2012). Furthermore, the cluster structure also has a low dependency to the polarization variation of the incident light, which simplifies the experimental condition, making it a more efficient enhancement system.

In this paper, different agglomeration types of $\mathrm{Au}-\mathrm{Ag}$ alloy NPs are employed to investigate the enhancement effect of the fluorescence and Raman signal. Three different thickness levels of the $\mathrm{SiO}_{2}$ layer are coated on the alloy NPs and clusters to explore the optimized enhancement system for the fluorescence and Raman signal, respectively. The power dependence of the fluorescence and Raman signal are also studied on [Au-Ag alloy NP cluster] $@ \mathrm{SiO}_{2}$ core-shell nanostructure with different shell thickness. This work offers a convenient nonpolarization dependent $\mathrm{Au}-\mathrm{Ag}$ alloy cluster system to enhance both the fluorescence emission and Raman scattering, which have the promising application potential in portable chemical or biochemistry detecting.

\section{EXPERIMENT}

Sodium citrate reduction method (Lee and Meisel, 1982) are used in synthesizing citrate-reduced $\mathrm{Au}-\mathrm{Ag}$ alloy NPs. First, Aqueous solution of $\mathrm{AgNO}_{3}$ (99.8\%) and $\mathrm{HAuCl}_{4} \cdot 4 \mathrm{H}_{2} \mathrm{O}$, (99\%) are added in proportion into boiled deionized water and keeping reaction for $10 \mathrm{~min}$, then the reductant Sodium citrate (99\%) is added and heating is continued for $15 \mathrm{~min}$. Finally, isolated alloy NPs can be obtained after centrifugation $(8,000 \mathrm{r} / \mathrm{min})$ and washing with deionized water. We can get the alloy clusters by washing with ethyl alcohol and increasing the $\mathrm{rpm}(12,000 \mathrm{r} / \mathrm{min})$ during a repeating washing-centrifuging cycle. It should be noted that centrifugal speed and washing solution need to be controlled in order to control the dispersion of the obtained NPs.

The modified Stöber method (Lu et al., 2002; Lessard-Viger et al., 2009) is used to coat the $\mathrm{SiO}_{2}$ shell on Au-Ag alloy NPs or clusters. The obtained Au-Ag alloy NPs (clusters) are dissolved in isopropyl alcohol (99.7\%) and heated to $40^{\circ} \mathrm{C}$. Then deionized water, ammonium hydroxide $(25 \%)$ and tetraethyl orthosilicate (28.5\%) are added into the colloid and keeping reaction for $3.5 \mathrm{~h}$ with continuous stirring. The amount of silica precursor (tetraethyl orthosilicate) is important to the thickness of the silica shell. After washing with ethanol and deionized water, the obtained core-shell structures are dispersed in deionized water and mixed with probe molecules (Rh6G). Then the mixture solution after ultrasonic agitation was left to stand in the dark for 2 days at room temperature, in order to achieve a uniformly coated fluorophore molecule on the surface of the shell. The excess molecule in the solution can be removed by washing several times.

\section{RESULTS AND DISCUSSION}

With different ratios of the $\mathrm{Au}$ and $\mathrm{Ag}$ content, the SPR peak can be easily tuned from 400 to $530 \mathrm{~nm}$ (Figure 1A). The 1:1 ratio of the $\mathrm{Au}$ and $\mathrm{Ag}$ is chosen in the following parts, and corresponding elemental distribution is obtained through the energy dispersive X-ray (EDX) elemental mapping. As shown in Figures 1B-D, the elemental mapping image (Figures 1B-D) of $\mathrm{Au}-\mathrm{Ag}$ alloy NPs shows that the ratio of the $\mathrm{Au}$ and $\mathrm{Ag}$ content is 1:1. Besides, the morphology of the obtained isolated $\mathrm{Au}-\mathrm{Ag}$ alloy NPs, $\mathrm{Au}-\mathrm{Ag}$ alloy NP clusters, [isolated $\mathrm{Au}-\mathrm{Ag}$ alloy NP]@SiO 2 and $\left[\mathrm{Au}-\mathrm{Ag}\right.$ alloy NP cluster]@SiO $\mathrm{S}_{2}$ core-shell structure are characterized with TEM. As shown in Figure 2A, the uniform-sized Au-Ag alloy isolated nanospheres are synthesized with high dispersity. The thickness controllable $\mathrm{SiO}_{2}$ layer can be evenly coated on the surface of the Au-Ag alloy nanospheres (Figure 2B). Figure 2C shows the morphology of the Au-Ag alloy cluster which is composed with the uniform sized $\mathrm{Au}-\mathrm{Ag}$ alloy nanospheres, and corresponding $\mathrm{SiO}_{2}$ coated structure is shown in Figure 2D. Meanwhile, the different thickness of the $\mathrm{SiO}_{2}$ layer can also be selectively coated on the cluster.

In order to evaluate the ability of the enhancement on Raman scattering and fluorescence emission of different system, Rh6G molecule is chosen as a fluorescence/Raman detecting molecule, which is uniformly absorbed on the surface of $\mathrm{SiO}_{2}$ layer. Here we choose [isolated Au-Ag alloy NP] @SiO $\mathrm{S}_{2}$ system (Figure 2A) to compare with the [Au-Ag alloy NP cluster]@SiO ${ }_{2}$ system (Figure 2C). Both above systems are coated with Rh6G in same concentration. Surface enhanced Raman scattering (SERS) and surface enhanced fluorescence (SEF) spectra were investigated with quantitative core-shell particles system. The number of molecules absorbed on the surface of the $\mathrm{SiO}_{2}$ is different due to the different surface area. Thus, all the spectra are normalized with the surface area, and signal intensity is from the per unit area of the surface.

Three different levels of thickness $(2,8$, and $15 \mathrm{~nm})$ of $\mathrm{SiO}_{2}$ shell are selectively chosen to investigate the sensitivity of SERS/SEF effect. SEF spectra of single [isolated Au-Ag alloy $\mathrm{NP}] @ \mathrm{SiO}_{2}$ (Figure 3A) and multiple tightly distributed shell isolated core-shell NPs (Figure 3B) with different shell thickness is shown in Figures 3C,D. It is obvious that the fluorescence signal is effectively enhanced in the core-shell system, especially for the $8 \mathrm{~nm} \mathrm{SiO} 2$ coated system. However, no obvious SERS signal could be detected in both two spectra, which means that the Raman scattering cannot be effectively enhanced and observed by the above single Au-Ag alloy $\mathrm{NPs@SiO}_{2}$ shell isolated NP or multiple tightly distributed shell isolated coreshell NPs.

Figure 4 shows the enhancement effect of Raman and fluorescence signal of the [Au-Ag alloy NP cluster]@SiO $\mathrm{S}_{2}$ system. As shown in Figures 4A-C, three different thickness levels of the 


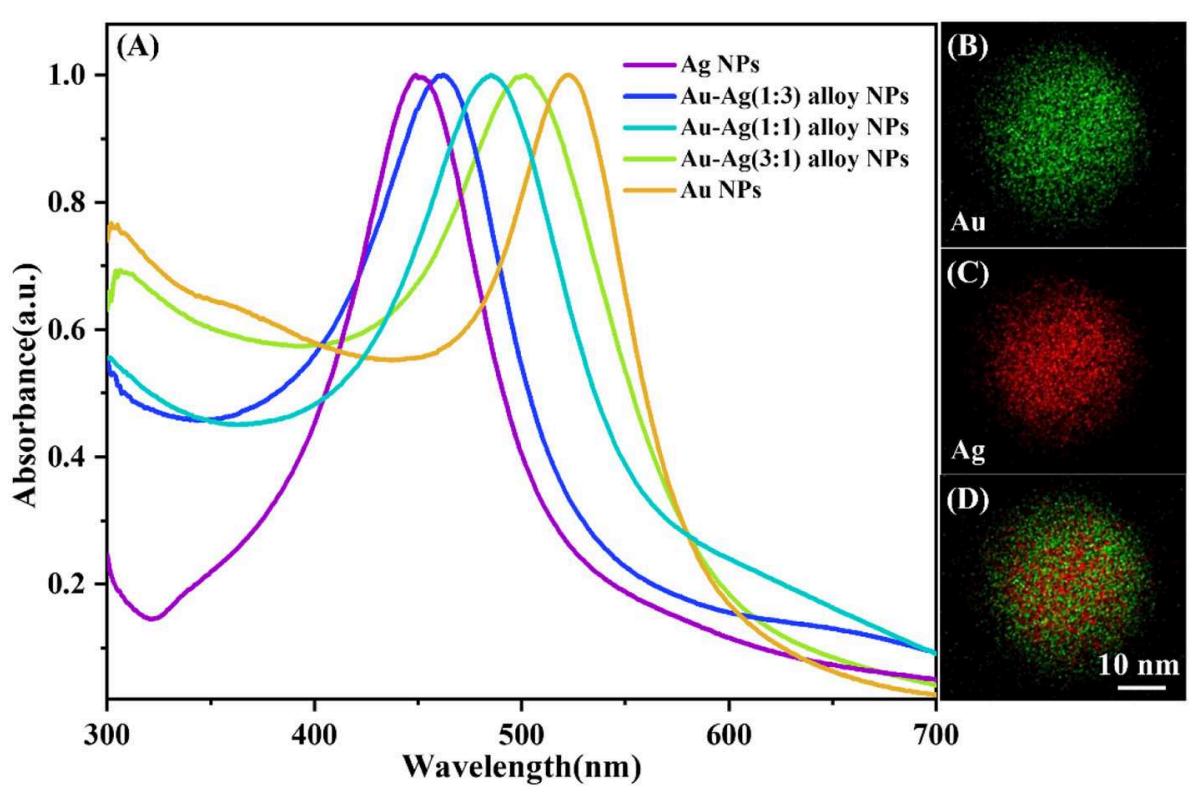

FIGURE 1 | (A) Absorption spectra of pure Au and Ag nanospheres, and bimetallic Au-Ag alloy nanospheres with different ratio of gold to silver 1:3, 1:1, and 3:1. STEM-EDX elemental mapping image of Au-Ag alloy NP with the ratio of gold to silver 1:1, where the green represents Au (B), red represents Ag (C), and the merged image (D) from single Au-Ag alloy nanoparticle.
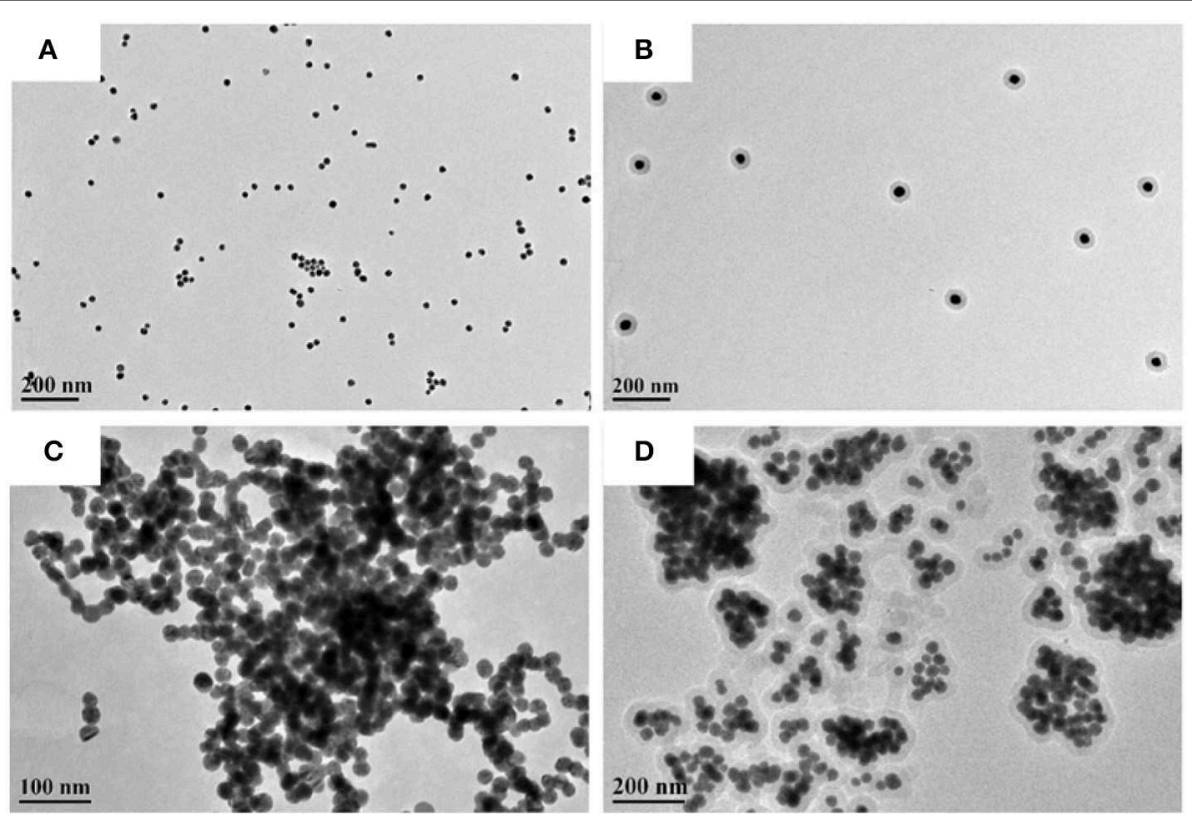

FIGURE 2 | TEM images of (A) isolated Au-Ag alloy NPs. (B) [isolated Au-Ag alloy NP]@SiO2. (C) Au-Ag alloy NP cluster. (D) [Au-Ag alloy NP cluster]@SiO 2 .

$\mathrm{SiO}_{2}$ layer $(2,8$, and $15 \mathrm{~nm})$ are evenly coated on the $\mathrm{Au}-\mathrm{Ag}$ alloy cluster. Figures $4 \mathrm{D}-\mathrm{F}$ show the power-dependence in-situ spectra of the corresponding samples shown in Figures 4A-C. When the thickness of the $\mathrm{SiO}_{2}$ is $2 \mathrm{~nm}$ (Figure 4D), both Raman and fluorescence signal are obviously enhanced by the system. As the laser power decreases, the intensity of the Raman scattering and fluorescence emission have obvious damping, but the Raman signal can still be clearly observed under the lowest power excitation, which results in the high intensity EM field generated by the alloy cluster. When comparing to the spectra of Figures $4 \mathrm{E}, \mathrm{F}$ that were obtained from the cluster coated with thicker $\mathrm{SiO}_{2}$, the fluorescence intensity of the cluster 

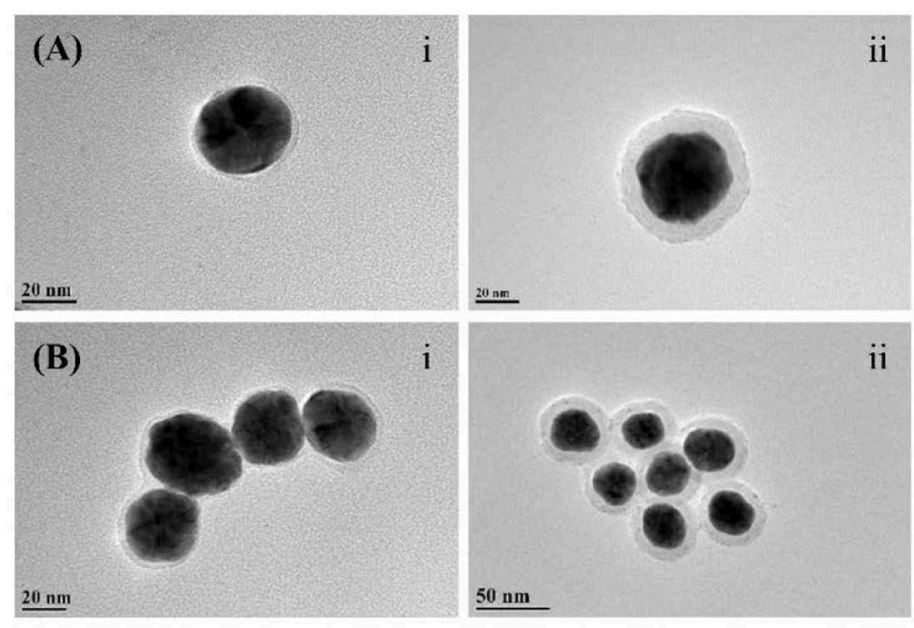
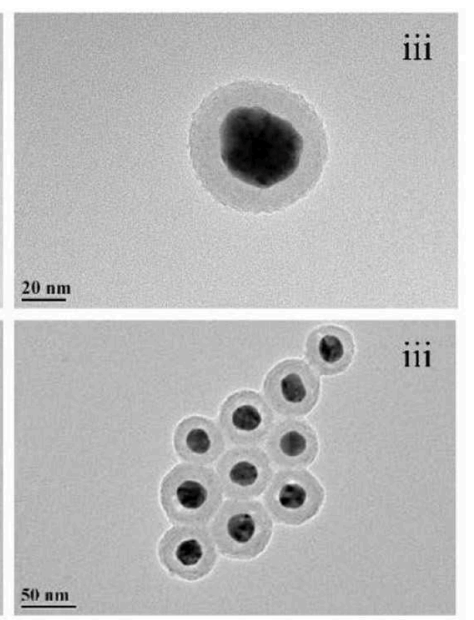
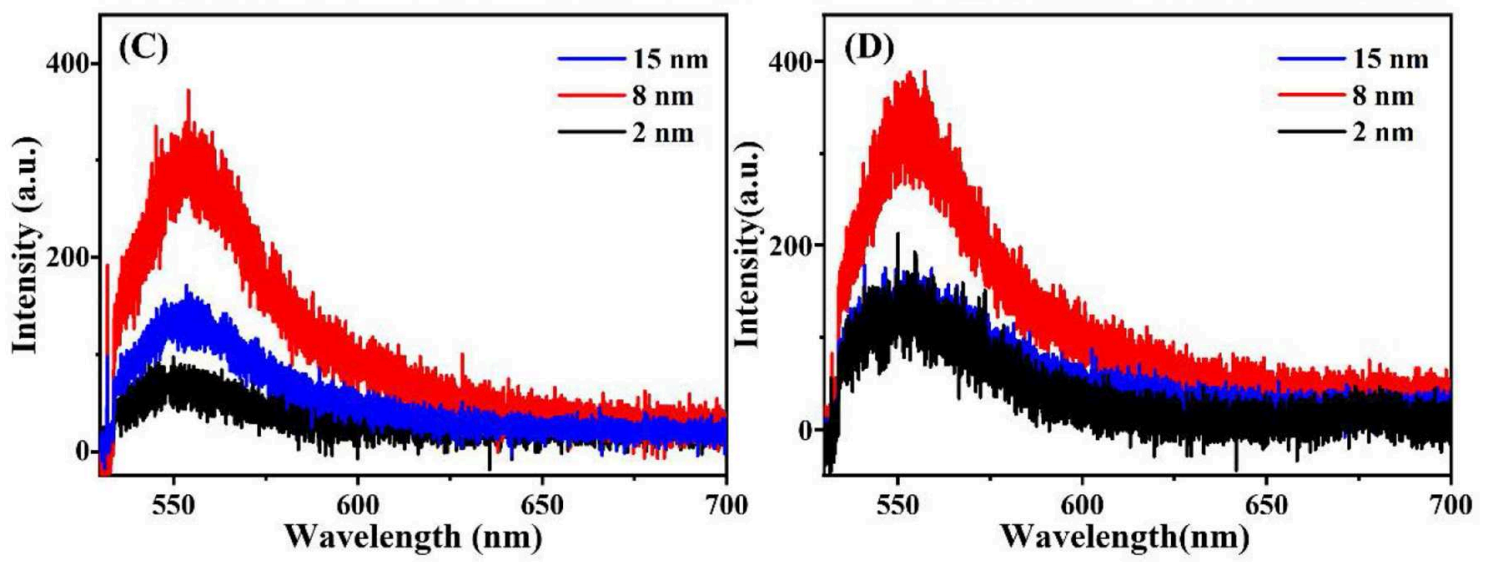

FIGURE 3 | TEM images and SEF spectra of Au-Ag alloy@SiO 2 shell isolated system with different thickness of $\mathrm{SiO}_{2}$ layer. (A) [lsolated Au-Ag alloy NP]@SiO ${ }_{2}$ with the $\mathrm{SiO}_{2}$ layer of $2 \mathrm{~nm}$ (i); $8 \mathrm{~nm}$ (ii); $15 \mathrm{~nm}$ (iii), respectively. (B) Aggregated [isolated Au-Ag alloy NP]@SiO $\mathrm{N}_{2}$ core-shell structure with the SiO 2 layer of $2 \mathrm{~nm}$ (i); $8 \mathrm{~nm}$ (ii); $15 \mathrm{~nm}$ (iii), respectively. (C) SEF spectra of the single [isolated Au-Ag alloy NP]@ $\mathrm{SiO}_{2}$ core-shell NP with different $\mathrm{SiO} 2$ layer shown in (A). (D) SEF spectra of a certain number of closely arranged core-shell NPs with different $\mathrm{SiO}_{2}$ layer shown in (B).

coated with $2 \mathrm{~nm} \mathrm{SiO}_{2}$ is obviously lower under the same power laser excitation, which is attributed to the quenching effect of SPR. When the molecules are located in the vicinity of the plasmonic metal NPs, the fluorescence emission intensity will be affected by many factors, such as the enhanced local field and the energy transfer between the molecule and plasmonic metal and the field. In general, the local EM field brings with it the enhancement effect of the excitation of molecules, while the effect of energy exchange between metal and molecules on SEF will change from quenching to enhancement with an increase of spacing (Zhang et al., 2016). The EM fields are confined at the surface of the shell, as its spatially inhomogeneous distribution is dramatically decayed with increased thickness of the $\mathrm{SiO}_{2}$, and the overall SEF results is dependent on the competition effects between the excitation enhancement and quenching effect. Alloy NP cluster coated with $8 \mathrm{~nm} \mathrm{SiO}_{2}$ shows the best enhancement effect on the fluorescence of the Rh6G molecular (Figure 4E). For the optimized shell thickness ( $8 \mathrm{~nm}$ ) of SEF, it is interesting that the SERS signal cannot be observed under the relative high-power excitation, due to the ultra-high enhanced fluorescence emission that may cover the SERS signal. Thus, it can be seen that the Raman scattering can only be obviously observed between the power of $0.9 \times 10^{5}$ and 3 $\times 10^{5} \mathrm{~W}$ with the $8 \mathrm{~nm}$ shell. Moreover, as for the [Au-Ag alloy NP cluster]@SiO 2 with $15 \mathrm{~nm}$ silica layer, because the EM enhancement is too weak at the surface of shell far away from the cluster, both the Raman and fluorescence signal cannot be effectively enhanced.

Comparing the influence of the $\mathrm{SiO}_{2}$ layer to the SERS/SEF intensity under different power excitation, Raman scattering and fluorescence emission intensity of the Rh6G molecules are obtained from the per unit area of the molecules adsorbed on the surface of the $\mathrm{SiO}_{2}$. Figure 5 shows the relation between the fluorescence (Raman) intensity and the laser power of different thickness of $\mathrm{SiO}_{2}$ coated Au-Ag NP cluster. As shown in Figure 5A, due to the fluorescence quenching effect with thin shell $(2 \mathrm{~nm})$ and the far distance with the EM enhanced field with thicker shell $(15 \mathrm{~nm})$, Au-Ag alloy NP 

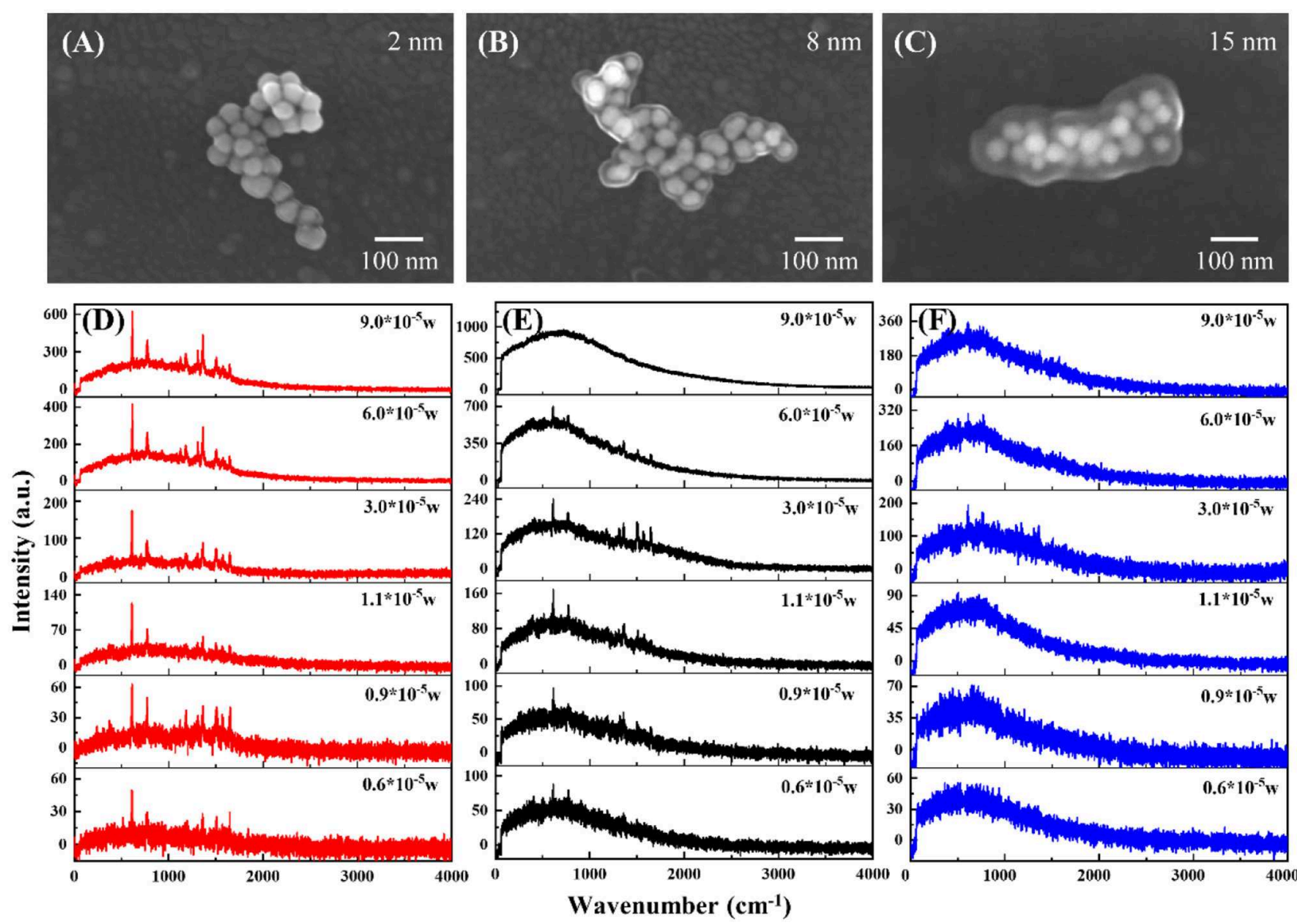

FIGURE 4 | SEM images of [Au-Ag alloy NP cluster]@SiO 2 core-shell nanostructure with the $\mathrm{SiO}_{2}$ layer of $2 \mathrm{~nm}$ (A), $8 \mathrm{~nm}$ (B) and $15 \mathrm{~nm}$ (C), respectively. Raman spectra of [Au-Ag alloy NP cluster] @SiO 2 under different laser power excitation with the $\mathrm{SiO}_{2}$ layer of $2 \mathrm{~nm}$ (D), $8 \mathrm{~nm}$ (E) and $15 \mathrm{~nm}$ (F), respectively.
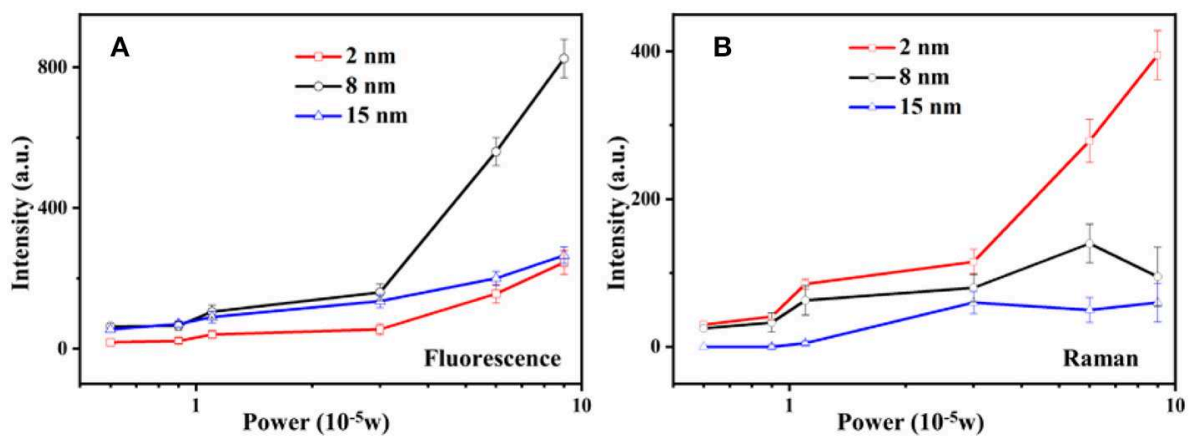

FIGURE 5 | Power dependence of the intensity of SEF (A) and SERS (B) with [Au-Ag alloy NP cluster]@SiO 2 core-shell structure.

cluster coated with $8 \mathrm{~nm}$ silica shell is the optimized system for the fluorescence enhancement. Figure 5B indicates the relation between the Raman intensity and the laser power. Different from the fluorescence emission, the intensity of Raman scattering demonstrates a positive correlation to the intensity of the biquadrate of the EM field $\left(|E|^{4}\right)$. Thus, NP cluster coated with $2 \mathrm{~nm}$ silica shell shows the great performance in SERS.
Nevertheless, in core-shell structure, to get the high quality of the SERS signal, the influence of fluorescence has to be excluded due to the fluorescence signal coverage to the Raman signal. The alloy NP cluster coated with $8 \mathrm{~nm}$ shows a great performance in fluorescence enhancement, which means it is unsuitable for the SERS enhancement system. The anticlimactic intensity of the Raman scattering at the largest power excitation, shown as the 
black line, results in the competition between the fluorescence and Raman signal.

\section{CONCLUSION}

Isolated Au-Ag alloy NPs and Au-Ag alloy NP clusters are employed to investigate and compare the enhancement effect of the fluorescence and Raman signal. Rh6G molecular with the same concentration are absorbed on three different thickness levels of the $\mathrm{SiO}_{2}$ layer which is coated on the Au-Ag alloy NPs and clusters. Under the same power laser excitation, the single [isolated Au-Ag alloy NP]@SiO 2 particle and their aggregates cannot effectively enhance the Raman scattering of the adsorbed molecular. However, as seen from the strong near-field enhancement of the alloy NP cluster, both 2 and $8 \mathrm{~nm} \mathrm{SiO} 2$ coated cluster system can obviously enhance the Raman signal, and Au-Ag alloy NP cluster with $8 \mathrm{~nm}$ silica shell shows the best performance in fluorescence enhancement. This work offers a convenient non-polarization dependent Au-Ag alloy NP cluster system to enhance both the fluorescence and Raman signal,

\section{REFERENCES}

Adams, S. M., Campione, S., Caldwell, J. D., Bezares, F. J., Culbertson, J. C., Capolino, F., et al. (2012). Non-lithographic SERS substrates: tailoring surface chemistry for Au nanoparticle cluster assembly. Small 8, 2239-2249. doi: 10.1002/smll.201102708

Ando, J., Fujita, K., Smith, N. I., and Kawata, S. (2011). Dynamic SERS imaging of cellular transport pathways with endocytosed gold nanoparticles. Nano Lett. 11, 5344-5348. doi: $10.1021 / \mathrm{nl} 202877 \mathrm{r}$

Chen, Y., and Ming, H. (2012). Review of surface plasmon resonance and localized surface plasmon resonance sensor. Photonic Sens. 2, 37-49. doi: 10.1007/s13320-011-0051-2

Christopher, P., Xin, H., and Linic, S. (2011). Visible-light-enhanced catalytic oxidation reactions on plasmonic silver nanostructures. Nat. Chem. 3:467. doi: $10.1038 /$ nchem.1032

Ding, S.-Y., Yi, J., Li, J.-F., Ren, B., Wu, D.-Y., Panneerselvam, R., et al. (2016). Nanostructure-based plasmon-enhanced Raman spectroscopy for surface analysis of materials. Nat. Rev. Mater. 1:16021. doi: 10.1038/natrevmats. 2016.21

Gao, C., Hu, Y., Wang, M., Chi, M., and Yin, Y. (2014). Fully alloyed $\mathrm{Ag} / \mathrm{Au}$ nanospheres: combining the plasmonic property of $\mathrm{Ag}$ with the stability of Au. J. Am. Chem. Soc. 136, 7474-7479. doi: 10.1021/ja50 $2890 \mathrm{c}$

Han, L., Li, C., Zhang, T., Lang, Q., and Liu, A. (2015). Au@Ag heterogeneous nanorods as nanozyme interfaces with peroxidase-like activity and their application for one-pot analysis of glucose at nearly neutral $\mathrm{pH}$. ACS Appl. Mater. Interfaces 7, 14463-14470. doi: 10.1021/acsami.5b 03591

Han, Q., Zhang, C., Gao, W., Han, Z., Liu, T., Li, C., et al. (2016). Ag-Au alloy nanoparticles: synthesis and in situ monitoring SERS of plasmonic catalysis. Sens. Actu. B Chem. 231, 609-614. doi: 10.1016/j.snb.2016. 03.068

Hao, F., Nehl, C. L., Hafner, J. H., and Nordlander, P. (2007). Plasmon resonances of a gold nanostar. Nano Lett. 7, 729-732. doi: 10.1021/nl062969c

Homola, J., Yee, S. S., and Gauglitz, G. (1999). Surface plasmon resonance sensors: review. Sens. Actu. B Chem. 54, 3-15. doi: 10.1016/S0925-4005(98)00321-9

Kuladeep, R., Jyothi, L., Alee, K. S., Deepak, K. L. N., and Rao, D. N. (2012). Laserassisted synthesis of Au-Ag alloy nanoparticles with tunable surface plasmon resonance frequency. Opt. Mater. Exp. 2, 161-172. doi: 10.1364/OME.2.000161

Lee, P., and Meisel, D. (1982). Adsorption and surface-enhanced Raman of dyes on silver and gold sols. J. Phys. Chem. 86, 3391-3395. which have promising application potentials in portable chemical or biochemical detection.

\section{DATA AVAILABILITY STATEMENT}

All datasets generated for this study are included in the manuscript/supplementary files.

\section{AUTHOR CONTRIBUTIONS}

$\mathrm{CZ}$ and $\mathrm{HZ}$ conceived the idea. $\mathrm{CZ}$ did the experiment and the measurement. $\mathrm{CZ}$ and $\mathrm{TZ}$ discussed and analyzed the results. TZ and $\mathrm{CZ}$ wrote the paper. All the authors revised the paper.

\section{FUNDING}

This work was supported by the National Natural Science Foundation of China (Grant 11574190), the Fundamental Research Funds for the Central Universities (GK201701008, No. 2017TS013, 2018CSLZ001).
Lessard-Viger, M., Rioux, M., Rainville, L., and Boudreau, D. (2009). FRET enhancement in multilayer core-shell nanoparticles. Nano Lett. 9, 3066-3071. doi: $10.1021 / \mathrm{nl} 901553 \mathrm{u}$

Li, C., Wang, C., Han, Q., Wu, Y., and Zheng, H. (2017). Investigation on optical properties of Ag-Au alloy nanoparticles. Plasmonics 12, 1373-1379. doi: 10.1007/s11468-016-0396-x

Li, J. F., Huang, Y. F., Ding, Y., Yang, Z. L., Li, S. B., Zhou, X. S., et al. (2010). Shell-isolated nanoparticle-enhanced Raman spectroscopy. Nature 464:392. doi: $10.1038 /$ nature08907

Liu, A., Wang, G., Wang, F., and Zhang, Y. (2017). Gold nanostructures with nearinfrared plasmonic resonance: Synthesis and surface functionalization. Coord. Chem. Rev. 336, 28-42. https://doi.org/10.1016/j.ccr.2016.12.019

Liu, S., Chen, G., Prasad, P. N., and Swihart, M. T. (2011). Synthesis of Monodisperse $\mathrm{Au}, \mathrm{Ag}$, and $\mathrm{Au}-\mathrm{Ag}$ Alloy nanoparticles with tunable size and surface plasmon resonance frequency. Chem. Mater. 23, 4098-4101. doi: $10.1021 / \mathrm{cm} 201343 \mathrm{k}$

Lu, Y., Yin, Y., Mayers, B. T., and Xia, Y. (2002). Modifying the surface properties of superparamagnetic iron oxide nanoparticles through a sol-gel approach. Nano Lett. 2, 183-186. doi: 10.1021/nl015681q

Mia, X., Wang, Y., Li, R., Sun, M., Zhang, Z., and Zheng, H. (2019). Multiple surface plasmon resonances enhanced nonlinear optical microscopy. Nanophotonics 8, 487-493. doi: 10.1515/nanoph-201 8-0231

Ming, T., Zhao, L., Yang, Z., Chen, H., Sun, L., Wang, J., et al. (2009). Strong polarization dependence of plasmon-enhanced fluorescence on single gold nanorods. Nano Lett. 9, 3896-3903. doi: 10.1021/nl902095q

Nie, S., and Emory, S. R. (1997). Probing single molecules and single nanoparticles by surface-enhanced raman scattering. Science 275, 1102-1106. doi: $10.1126 /$ science.275.5303.1102

Rioux, D., Vallières, S., Besner, S., Muñoz, P., Mazur, E., and Meunier, M. (2014). An analytic model for the dielectric function of $\mathrm{Au}, \mathrm{Ag}$, and their Alloys. $\mathrm{Adv}$. Opt. Mater. 2, 176-182. doi: 10.1002/adom.201300457

Rosman, C., Prasad, J., Neiser, A., Henkel, A., Edgar, J., and Sönnichsen, C. (2013). Multiplexed plasmon sensor for rapid label-free analyte detection. Nano Lett. 13, 3243-3247. doi: 10.1021/nl401354f

Schietinger, S., Aichele, T., Wang, H.-Q., Nann, T., and Benson, O. (2010). Plasmon-enhanced upconversion in single $\mathrm{NaYF} 4: \mathrm{Yb}^{3+} / \mathrm{Er}^{3+}$ codoped nanocrystals. Nano Lett. 10, 134-138. doi: 10.1021/nl903046r

Sherry, L. J., Chang, S.-H., Schatz, G. C., Van Duyne, R. P., Wiley, B. J., and Xia, Y. (2005). Localized surface plasmon resonance spectroscopy of single silver nanocubes. Nano Lett. 5, 2034-2038. doi: 10.1021/nl0515753 
Talley, C. E., Jackson, J. B., Oubre, C., Grady, N. K., Hollars, C. W., Lane, S. M., et al. (2005). Surface-enhanced raman scattering from individual Au nanoparticles and nanoparticle dimer substrates. Nano Lett. 5, 1569-1574. doi: $10.1021 / \mathrm{nl} 050928 \mathrm{v}$

Tsukamoto, D., Shiro, A., Shiraishi, Y., Sugano, Y., Ichikawa, S., Tanaka, S., et al. (2012). Photocatalytic $\mathrm{H}_{2} \mathrm{O}_{2}$ production from Ethanol/O $\mathrm{O}_{2}$ system using $\mathrm{TiO}_{2}$ Loaded with $\mathrm{Au}-\mathrm{Ag}$ bimetallic alloy nanoparticles. ACS Catal. 2, 599-603. doi: $10.1021 / \operatorname{cs} 2006873$

Vadai, M., Angell, D. K., Hayee, F., Sytwu, K., and Dionne, J. A. (2018). In-situ observation of plasmon-controlled photocatalytic dehydrogenation of individual palladium nanoparticles. Nat. Commun. 9:4658. doi: 10.1038/s41467-018-07108-x

Wang, F., Liu, P., Sun, L., Li, C., Petrenko, V. A., and Liu, A. (2014). Bio-mimetic nanostructure self-assembled from Au@Ag heterogeneous nanorods and phage fusion proteins for targeted tumor optical detection and photothermal therapy. Sci. Rep. 4:6808. doi: 10.1038/srep06808

Wang, X. A., and Kong, X. (2015). Review of recent progress of plasmonic materials and nano-structures for surface-enhanced raman scattering. Materials 8, 3024-3052. doi: 10.3390/ma8063024
Zhang, C., Han, Q., Li, C., Zhang, M., Yan, L., and Zheng, H. (2016). Metalenhanced fluorescence of single shell-isolated alloy metal nanoparticle. Appl. Opt. 55, 9131-9136. doi: 10.1364/AO.55.009131

Zhang, X., Li, X., Reish, M. E., Zhang, D., Su, N. Q., Gutiérrez, Y., et al. (2018). Plasmon-enhanced catalysis: distinguishing thermal and nonthermal effects. Nano Lett. 18, 1714-1723. doi: 10.1021/acs.nanolett.7b 04776

Conflict of Interest: The authors declare that the research was conducted in the absence of any commercial or financial relationships that could be construed as a potential conflict of interest.

Copyright (C) 2019 Zhang, Zhang, Zhang and Zheng. This is an open-access article distributed under the terms of the Creative Commons Attribution License (CC BY). The use, distribution or reproduction in other forums is permitted, provided the original author(s) and the copyright owner(s) are credited and that the original publication in this journal is cited, in accordance with accepted academic practice. No use, distribution or reproduction is permitted which does not comply with these terms. 